\title{
Was the COVID-19 pandemic avoidable? A call for a "solution-oriented" approach in pathogen evolutionary ecology to prevent future outbreaks
}

\author{
ROCHE Benjamin ${ }^{1}$, Andres Garchitorena ${ }^{2}$, Jean-Francois Guegan ${ }^{2}$, Audrey Arnal², Serge \\ Morand $^{2}$, Gerardo Suzan ${ }^{2}$, David Roiz ${ }^{2}$, Carlos Zambrana-Torrelio ${ }^{2}$, and Peter Daszak ${ }^{2}$ \\ ${ }^{1}$ Institut de recherche pour le développement \\ ${ }^{2}$ Affiliation not available
}

August 6, 2020

\begin{abstract}
Concerns about the prospect of a global pandemic have been triggered many times during the last two decades. These have been realized through the current COVID-19 pandemic, due to a new coronavirus SARS-CoV2, which has impacted almost every country on Earth. Here, we show how considering the pandemic through the lenses of the evolutionary ecology of pathogens can help better understand the root causes and devise solutions to prevent the emergence of future pandemics. We call for better integration of these approaches into transdisciplinary research and invite scientists working on the evolutionary ecology of pathogens to contribute to a more "solution-oriented" agenda with practical applications, emulating similar movements in the field of economics in recent decades.
\end{abstract}

The COVID-19 pandemic, caused by SARS-CoV-2, has produced a major global crisis, infected millions and killed hundreds of thousands throughout the world, collapsed health systems, generated social disruptions and paralyzed the global economy ${ }^{1}$. This unprecedented worldwide outbreak has consequences that are hard to predict but at least could be comparable to the 1918 influenza pandemic in terms of socio-economic and political impacts on our global society ${ }^{2}$. Societies are already adjusting to the fact that the world after the COVID-19 pandemic will be extremely different.

Scientists from multiple disciplines and public health experts have been mobilized across the globe to support policy makers and design strategies to mitigate as much as possible the impacts of COVID-19. Efforts have included the coordination and prompt release of underlying data, open discussions of different methodological approaches and sharing results to accelerate medicine and research discoveries. This inter-disciplinary and international effort is without precedent and shows that the scientific community can be rapidly mobilized to provide solutions and answers to urgent issues during global crises. Importantly, at a time of great uncertainty, many governments are unusually receptive to scientific inputs to inform evidence-based decision making, which is a positive shift in the midst of a difficult situation.

Yet, an event such as the current COVID-19 pandemic was a foregone conclusion. The rate of appearance of new emerging infectious diseases (EIDs), most of them with a wildlife origin, has increased in past decades ${ }^{3}$ and recent outbreaks including $\mathrm{H} 5 \mathrm{N1}^{4}$, MERS-CoV ${ }^{5}$, Zika ${ }^{6}$ and Ebola ${ }^{7}$ have previously raised concerns over their potential to cause pandemics. These outbreaks were controlled partly because their transmissibility and virulence made them more amenable to public health control measures. However, scientists and public health experts have been raising warnings for years about the potential for contagious avian influenza or coronavirus spillovers (among others) ${ }^{8}$ to become the next global pandemic ${ }^{9}$, as they are frequently found 
in both domestic (e.g., pigs, cats, bovines, birds) and wild animals (e.g., bats, pangolins). They have also repeatedly shown their ability to infect humans through multiple spillover events that have led to humanto-human transmission.

\section{But do we know why this happened? In other words, was this pandemic avoidable and are we ready to prevent or contain the inevitable next one?}

SARS-CoV-2 is the perfect example of a zoonosis spilling over from wildlife and establishing shortly afterwards in human populations. This type of event has happened many times during human evolution and in the history of human civilizations ${ }^{10}$. For example, diseases such as smallpox, bubonic plague, and cholera, introduced by Europeans to the Americas and Africa caused extinction of indigenous communities, but had initially an animal origin. Other diseases such as yellow fever, HIV/AIDS, dengue and Zika all emerged from non-human primates. Similarly, measles diverged from rinderpest, a pathogen that has circulated in livestock for centuries with now humans being the only reservoir. While this type of emergence is not new, we now live in a world that is more connected than ever due to the globalization of trade, advances in transportation systems, a population experiencing exponential growth and the highest rates of urbanization in history. Connectivity clearly accelerates disease spread once a spillover event has occurred, but the frequency and volume of those events is also increasing due to global environmental changes.

A key contribution of evolutionary ecology is to help understand pathogen circulation within animal communities. Viruses of pandemic potential such as avian influenza and coronaviruses circulate within animal communities, infecting a great diversity of species including different species of birds, bats, canines, felids, rodents, equines, camelids, and rabbits ${ }^{11}$. The structure of these animal communities and their biological properties (e.g., species richness or species composition) can dramatically modulate pathogen emergence, the range of virus circulation and their evolutionary trajectories. While we are undergoing the $6^{\text {th }}$ mass extinction on record and the first due to anthropogenic pressures, some studies (despite controversies) suggest that a loss of biodiversity can increase transmission of some pathogens ${ }^{12}$.

Understanding the underlying drivers of pathogen spillover and the role of biodiversity in pathogen transmission can help devise proactive ecological interventions to prevent and reduce spillover events. Indeed, ecosystem management and other strategies to protect species diversity, including restoration, rewilding and the management of wildlife reserves, halting habitat loss, population declines and illegal wildlife trade, can be a lever to prevent and mitigate pathogen emergence and transmission. In addition, increasing rates of emergence from domestic animals can be attributed to the demographic explosion and genetic homogeneity derived from intensive farming systems, where they can act as amplifiers and edge-hosts of novel pathogens. Increasing host diversity within farming systems can moderate the role of domestic animals in the emergence of new pathogens, as has been shown for plant pathogens ${ }^{13}$.

An additional driver of emergence is the rising exposure of human populations to pathogens due to habitat encroachment and settlement into natural systems ${ }^{14}$. With nearly $40 \%$ of the Earth's land surface already converted to cropland or pasture, most of the land conversion in recent decades is taking place in forests of tropical and subtropical areas, which represent some of the greatest hotspots of biodiversity ${ }^{15}$. This increased exposure to previously isolated wildlife and their pathogens is amplified by human activities such as bushmeat consumption, recreational and agricultural activities, or wildlife trade. Reducing this animal/human interface can be partly achieved through law enforcement, education programs for at-risk groups to reduce their exposure to viral contamination, and by providing viable alternatives to bushmeat hunting, which is always associated with the poorest communities, in order to minimize human exposure to wildlife. However, with roughly two thirds of all agricultural land used for animal grazing and pasture, and a population expected to reach 9 billion people by 2050, swift changes in food production and consumption patterns will be needed to avoid further encroachment and settlement due to agricultural needs.

We know that this human-animal interface can potentially impact pathogen adaptation to human populations. So far, most of the medical research focused on this topic consists in finding genetic markers demon- 
strating that an adaptation has taken place and would yield an efficient human-human transmission ${ }^{16}$. However, fundamental research accumulated in evolutionary ecology can bring important insights to the subject by identifying how such adaptation to a new host can be promoted ${ }^{17}$. Such studies could therefore provide practical knowledge for pandemic risk management if they become rooted in real-world data (e.g., by considering contact data between human and animal species) and/or on experimental models that can mimic closely human/animal contact processes.

While we now have a substantial understanding of the ecological and evolutionary drivers of pathogen spillover, there is a critical knowledge gap regarding which ecological interventions will most effectively reduce human disease burdens and pandemic risk. Empirical examples exist, such as pilot interventions on prawn farms to control schistosomiasis via reduction of the snail intermediate host or using landscape management to modify bat distribution and reduce risk of bat-borne pathogens ${ }^{18,19}$. Nevertheless, these remain scarce ${ }^{18,19}$. In this sense, one of the greatest opportunities in disease ecology lies in integrating pathogen data collection and evaluation methods (e.g.,observational, quasi-experimental) with the myriad of ecosystem management and conservation efforts happening around the world.

Efforts to tackle many of these issues in order to reduce the likelihood of the next pandemic will face seemingly unsurmountable challenges as well as conflicting political and economic agendas. Yet, if the COVID-19 epidemic has taught us anything, it is that in a globalized world, decisive action, widespread behavioral changes and large investments to avoid catastrophic health consequences are not only possible but can happen over short time scales. In less than six months since the first cases were declared, roughly one third of the world's population has been forced to self-quarantine, whole economic sectors have been drawn to a halt and most international and domestic travel has stopped. G20 countries have already committed 5 trillion USD just to keep their economies afloat and are imposing restrictions that affect most aspects of their citizens' daily lives. The global recession is also revealing how reactive approaches might be orders of magnitude more expensive than proactive ones. These economic efforts should be redirected towards developing evidence-based preventive approaches in the post COVID-19 era through a globally coordinated interdisciplinary and integrated program focused on the improvement of current systems for pandemic risk prediction in the short-term, as well as identifying and testing long-term solutions in current and future socio-ecosystems that can quantitatively reduce the probability of new pandemics.

The case for investments in drug and vaccine development seems more straightforward and is certainly justified, especially as a pandemic is underway, but these should also be complemented with policies and strategies that address the root causes of disease emergence in global hotspots such as educational programs to reduce exposure to wildlife by Asian farmers or biological conservation in Latin America or sub-Saharan Africa. Though less visible, this preparedness strategy could certainly be highly efficient and cost-effective, especially if we consider the health and economic costs averted by a reduction in spillover events and a reduced likelihood of a new pandemic. Furthermore, investing in stronger health systems, universal access to healthcare and education in developing countries could help contain these outbreaks before they become widespread. It is therefore mandatory to develop integrative approaches that combine long-term pandemic prevention via ecosystem management with reinforced health systems for better disease surveillance and control, in collaboration with a social science perspective to improve our understanding of the barriers and the enablers for adoption of such solutions.

Governments are relying on scientists during this time of "war" against COVID-19, but such reliance is even more important during 'peace' time. We should not consider this pandemic as an isolated phenomenon, but rather as a part of a causal chain of events derived from our actions, i.e., a systemic crisis. There is an overwhelming scientific consensus that we are irreversibly deteriorating ecosystem integrity and the services they provide ${ }^{20}$, transgressing planetary boundaries and running out of time to take decisive action and reverse this trend ${ }^{21}$. Only by investing in scientific knowledge in close partnership with key stakeholders (e.g., local authorities and citizens, public health authorities, supra-national organizations, etc.) we will be able to find actionable solutions, guide policy decisions and avoid future catastrophes. As detailed previously, the 
need for ecological and evolutionary approaches to understand, model and predict pandemics in a changing world is crucial. Focusing our efforts only on emergency and mitigation measures against COVID-19 would constitute a major missed opportunity to combat the broader structural causes of the pandemic. The downstream consequences would be dramatic.

We call for an urgent consideration of ecological and evolutionary approaches to understand and model pandemics in a changing world in order to prevent them. Once again, we argue for transdisciplinary research conducted under the Ecohealth, OneHealth and Planetary Health frameworks. To date, these initiatives have been largely ignored by decision-makers compared to the purely medical perspective that relies instead on the development of curative methods to prevent or control pandemics. However, such frameworks can help us understand the connection between pathogen spread within human populations, inter-specific pathogen transmission within animal communities, and how this may lead to adaptation and emergence. The knowledge generated by this research can therefore greatly improve our capacity to prevent large-scale pandemics.

However, if such knowledge is not considered today by decision-makers, our scientific community could be blamed for not having put the knowledge produced into a "solution-oriented" framework that could be directly used by authorities. Therefore, we also call on scientists working on the evolutionary ecology of pathogens to integrate "solution-oriented" research, more connected to the field, in order to devise solutions and practical applications of scientific results, emulating recent similar movements in the field of economics and other social sciences 22. Biodiversity conservation, landscape management and theoretical evolution (among others) could all be "solution-oriented" research if applied to relevant pathogen models. Such "solution-oriented" research agendas could improve our capacity to avoid the next COVID19-like catastrophe.

\section{Acknowledgments:}

BR, GS and DR are supported by the French National Research Institute for Sustainable Development (IRD) through the international Joint Laboratory ELDORADO (Ecosystem, biological Diversity, habitat modifications, and Risk of emerging Pathogens and Diseases in Mexico). BR and J F G are supported by an 'Investissement d'Avenir' grant from the Agence Nationale de la Recherche (CEBA ANR-10-LABX-25-01). JFG is funded by a NSF Ecology of infectious diseases award \#191145. P.D. and C.Z.-T. acknowledges funding to EcoHealth Alliance from the Ford Foundation, The David and Lucile Packard Foundation and Johnson \& Johnson

Bibliography:

1. WHO. Coronavirus disease 2019 (COVID-19) Situation Report .available at https://www.who.int/emergencies/diseases/novel-coronavirus-2019 (2020).

2. Taubenberger, J. K., Kash, J. C. \& Morens, D. M. The 1918 influenza pandemic: 100 years of questions answered and unanswered. Sci. Transl. Med. (2019). doi:10.1126/scitranslmed.aau5485

3. Jones, K. E. et al. Global trends in emerging infectious diseases. Nature 451, 990-994 (2008).

4. Vijaykrishna, D. et al. Evolutionary dynamics and emergence of panzootic H5N1 influenza viruses. PLoS Pathog. (2008). doi:10.1371/journal.ppat.1000161

5. Cauchemez, S. et al. Unraveling the drivers of MERS-CoV transmission. PNAS (2016). doi:10.1073/pnas.1519235113

6. Musso, D., Ko, A. I. \& Baud, D. Zika virus infection - After the pandemic. New England Journal of Medicine (2019). doi:10.1056/NEJMra1808246 
7. Aylward, B. et al. Ebola virus disease in West Africa - The first 9 months of the epidemic and forward projections. N. Engl. J. Med. (2014). doi:10.1056/NEJMoa1411100

8. Cheng, V. C. C., Lau, S. K. P., Woo, P. C. Y. \& Kwok, Y. Y. Severe acute respiratory syndrome coronavirus as an agent of emerging and reemerging infection. Clinical Microbiology Reviews (2007). doi:10.1128/CMR.00023-07

9. WHO. Managing epidemics . (2018).

10. Lloyd-Smith, J. O. et al. Epidemic Dynamics at the Human-Animal Interface. Science (80-. ). 326 , 1362-1367 (2009).

11. Olival, K. J. et al. Host and viral traits predict zoonotic spillover from mammals. Nature (2017). doi:10.1038/nature22975

12. Ostfeld, R. S. \& Keesing, F. Effects of Host Diversity on Infectious Disease. Annu. Rev. Ecol. Evol. Syst. 43 , 157-182 (2012).

13. Jr, F. A. et al. Genetic diversity and disease control in rice. Nature 406 , 718-722 (2000).

14. Guégan, J.-F., Ayouba, A., Cappelle, J. \& de Thoisy, B. Forests and emerging infectious diseases: unleashing the beast within.Environ. Res. Lett. (2020). doi:10.1088/1748-9326/ab8dd7

15. Whitmee, S. et al. Safeguarding human health in the Anthropocene epoch: Report of the Rockefeller Foundation-Lancet Commission on planetary health. The Lancet (2015). doi:10.1016/S0140-6736(15)60901-1

16. Pepin, K. M., Lass, S., Pulliam, J. R. C., Read, A. F. \& Lloyd-Smith, J. O. Identifying genetic markers of adaptation for surveillance of viral host jumps. Nat Rev Microbiol 8 , 802-813 (2010).

17. Gandon, S., Of, E. \& Parasites, M. Evolution of Multihost Parasites. Evolution (N. Y). 58 , 455 (2004).

18. Sokolow, S. H. et al. Ecological interventions to prevent and manage zoonotic pathogen spillover. Philosophical Transactions of the Royal Society B: Biological Sciences (2019). doi:10.1098/rstb.2018.0342

19. Roche, B., Broutin, H. \& Simard, F. Ecology and Evolution of Infectious Diseases . 1 , (2018).

20. Kadykalo, A. N. et al. Disentangling 'ecosystem services' and 'nature's contributions to people'. Ecosystems and People (2019). doi:10.1080/26395916.2019.1669713

21. Steffen, W. et al. Planetary boundaries: Guiding human development on a changing planet. Science (80-. ). (2015). doi:10.1126/science.1259855

22. Duflo, E. \& Banerjee, A. V. Poor Economics: a radical rethinking on the way to fight . (2011). 